\title{
Dawson-type Polyoxometalate and Carbon nanotubes Nanocomposite for high-performance Supercapacitor Electrodes
}

\author{
Shuping LIU \\ College of Tourism and Cuisine, Harbin University of Commerce, Harbin 150076, China \\ email: liusp201@163.com
}

Keywords: Polyoxometalate; Carbon nanotubes; Layer-by-Layer; Electrochemical supercapacitors

\begin{abstract}
A nanocomposite film based on Dawson-type polyoxometalate cluster $\left(\mathrm{P}_{2} \mathrm{~W}_{18}\right)$ carbon nanotubes (CNTs) and chitosan (CS) were prepared on quartz and ITO substrates by layer-by-layer (LbL) method. These films were characterized by UV-vis spectroscopy, cyclic voltammetry (CV) and Scanning electron micrographs (SEM). It was shown that the multilayer film are uniform and stable. The multilayer film displays high supercapacitor performances by incorporation of CNTs into the $\mathrm{P}_{2} \mathrm{~W}_{18}$ film. The combined activity of $\mathrm{P}_{2} \mathrm{~W}_{18}$ and CNTs components in the composite film can store and release charge for electrochemical supercapacitors, leading to excellent specific capacitance value of $0.38 \mathrm{mF} \mathrm{cm}{ }^{-2}$.
\end{abstract}

\section{Introduction}

Electrochemical supercapacitors having higher power density than batteries and higher energy density than conventional capacitors are highly attractive energy-storage devices, and supercapacitors thus fill in the gap between batteries and conventional capacitors [1,2]. Recently, carbon-based materials are a kind of attractive electrode materials for supercapacitors application $[3,4]$. However, the carbon-based materials of in supercapacitors generally show poor charge-storage capacity. It is recognized that the fabrication of composite materials provides a useful technique for enhancing the charge-storage capacity.

Polyoxometalates (POMs) represent a well-known class of transition metal oxide nanoclusters with intriguing structures and diverse properties [5,6]. These interesting properties include molecular conductivity, reversible redox behavior, good chemical stability and multiple electron transfer, which make them attractive in areas such as catalysis, medicine, and material science $[7,8]$. The incorporation of POMs into the carbon-based materials produces composite materials that could present a more regular structure and higher electronic conductivity

In this paper, we successfully fabricate a nanocomposite film containing $\mathrm{P}_{2} \mathrm{~W}_{18}, \mathrm{CNTs}$, and chitosan (CS) by the layer-by-layer (LbL) assembly method. The composite film displays high supercapacitor performances by incorporation of CNTs into the $\mathrm{P}_{2} \mathrm{~W}_{18}$ film. The nanocomposite film is exploited as electrode materials for electrochemical supercapacitors, which shows improved specific capacitance values of $0.38 \mathrm{mF} \mathrm{cm}^{-2}$. These results can be helpful for exploring applications in electrochemical supercapacitors.

\section{Experimental details}

POM was prepared according to the literature method and identified by UV-vis absorption spectra. 3-Aminopropyltrimethoxysilane (APS), poly(styrenesulfonate) (PSS) (MW 70000) and poly (allylamine hydrochloride) (PAH) (MW 70000) were purchased from Aldrich and used without further treatment. CNTs were purchased from Tsinghua-Nafine Nano-power Commercialization Engineering Center. Other reagents were of AR grade. Before use, CNTs were treated with mixed acid according to a method already described and the oxidized CNTs (CNTs-COOH) were formed. Cs-CNTs composite was prepared by ultrasonication of $4 \mathrm{mg}$ of oxidized CNTs in $6 \mathrm{~mL}$ of $6 \mathrm{mg}$ chitosan in $0.1 \mathrm{~mol} / \mathrm{L}$ acetic acid.

Layer-by-layer films were assembled onto ITO-coated glass and quartz substrates. First, APS 
modified substrates were dipped into $\mathrm{HCl}(\mathrm{pH}=2.0)$ for $20 \mathrm{~min}$, and then immersed into PSS $(1 \times$ $\left.10^{-3} \mathrm{M} \mathrm{pH}=4.0\right)$ and PAH $\left(1 \times 10^{-3} \mathrm{M}\right)$ for $20 \mathrm{~min}$, respectively. Then the substrate-supported precursor films were alternately dipped into the $\mathrm{P}_{2} \mathrm{~W}_{18}\left(1 \times 10^{-3} \mathrm{M} \mathrm{pH}=4\right)$, CS-CNTs solutions for $8 \mathrm{~min}$ (referred to as $\left[\mathrm{P}_{2} \mathrm{~W}_{18}-\mathrm{CNTs}\right]_{\mathrm{n}}$ ). After deposition of each layer, the substrates were rinsed with deionized water after each dipping.

All the electrochemical experiments were performed on the CHI 660 electrochemical workstation (Shanghai Chenhua Instrument Factory, China). Scanning electron micrographs (SEM) were obtained with a Hitachi S-4800 instrument. Galvanostatic charge-discharge tests were carried out with three-electrode system. The electrolyte was the $0.1 \mathrm{~mol} / \mathrm{L} \mathrm{H}_{2} \mathrm{SO}_{4}$ aqueous solution. The specific capacitance was calculated according to the discharge part of curves.

\section{Results and discussion}

The composite film was assembled onto quartz and ITO-coated glass via ionic attraction of oppositely charged $\mathrm{P}_{2} \mathrm{~W}_{18}$ and CS-CNTs (see the inset in Fig. 1). UV-vis spectroscopy was used to monitor the layer-by-layer assembling process of $\mathrm{P}_{2} \mathrm{~W}_{18}$-CNTs composite films. Fig. 1 shows the transmittance spectra as a function of the number of $\left[\mathrm{P}_{2} \mathrm{~W}_{18}-\mathrm{CNTs}\right]_{\mathrm{n}}$ films (with $\mathrm{n}=1-6$ ) assembled on a quartz substrate. The composite films exhibit the characteristic transmittance of $\mathrm{P}_{2} \mathrm{~W}_{18}$ with bands at 198 and $280 \mathrm{~nm}$ in the UV region, which confirms the incorporation of $\mathrm{P}_{2} \mathrm{~W}_{18}$ into the composite films. The former is owing to the terminal oxygen to tungsten $\left(\mathrm{O}_{\mathrm{d}} \rightarrow \mathrm{W}\right)$ charge transfer transitions, and the latter is due to the charge transfer transitions from bridge-oxygen to tungsten $\left(\mathrm{O}_{\mathrm{b}} / \mathrm{O}_{\mathrm{c}} \rightarrow \mathrm{W}\right)$.

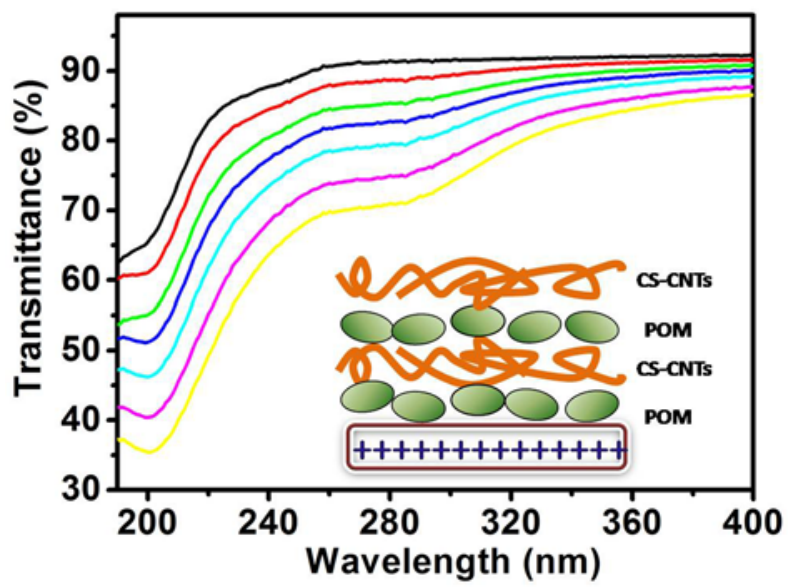

Fig. 1 UV-vis transmittance spectra of $\left[\mathrm{P}_{2} \mathrm{~W}_{18}-\mathrm{CNTs}\right]_{\mathrm{n}}$ films (with $\mathrm{n}=1-6$ ). Inset: schematics of self-assembly of a $\mathrm{P}_{2} \mathrm{~W}_{18}$-CNTs film.
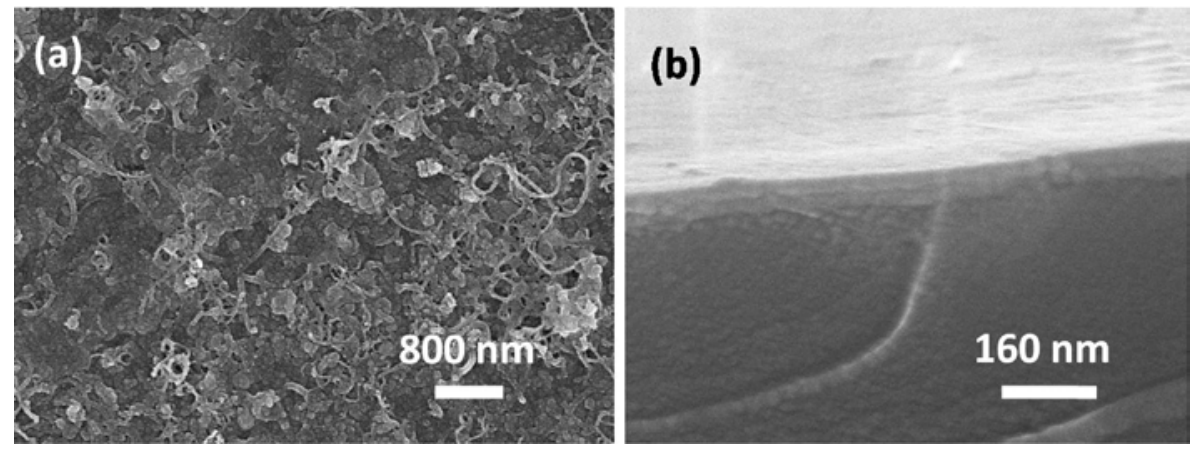

Fig. 2 SEM (a) and Cross-section (b) SEM image of a $\mathrm{P}_{2} \mathrm{~W}_{18}$-CNTs multilayer film on an ITO glass. 
SEM can provide the detailed information concerning the surface morphology and the homogeneity of the films. Before $\mathrm{P}_{2} \mathrm{~W}_{18}$-CNTs deposition, the precursor film is smooth. As shown in Fig. 2a, the film surface is relatively smooth over a large area and consists of a multitude of small domains which display a round shape. This perhaps arises from the absorption of bi- and multilayer aggregates of the $\mathrm{P}_{2} \mathrm{~W}_{18}$ polyanions and/or the CS chains. In addition, the hybrid nanowires caused by the interactions between CNTs and chitosan are clearly nanotube-like, suggesting the presence of CNTs. Fig. 2b displays the cross--section morphology of $\mathrm{P}_{2} \mathrm{~W}_{18}$-CNTs. From the cross-section observations, the composite film is fairly uniform.

To understand the electrochemical behavior of the $\mathrm{P}_{2} \mathrm{~W}_{18}-\mathrm{CNTs}$ film, the cyclic voltammograms $(\mathrm{CV})$ were measured in the potential range from -0.8 to $0.2 \mathrm{~V}$ at a scan rate of $50 \mathrm{mV} / \mathrm{s}$. A conventional three electrodes system was used, with the ITO electrode coated by the self-assembled film as the working electrode, $\mathrm{Ag} / \mathrm{AgCl}(3 \mathrm{M} \mathrm{KCl})$ as the reference electrode and platinum coil as the counter electrode. The $\mathrm{CV}$ of $\mathrm{P}_{2} \mathrm{~W}_{18}$ solution displays four redox peaks, which assigned to two 1-electron and two 2-electron redox processes (the inset of Fig. 3). As shown in Fig. 3, the films exhibit five couples of redox waves, which is similar to that of $\mathrm{P}_{2} \mathrm{~W}_{18}$ solution.

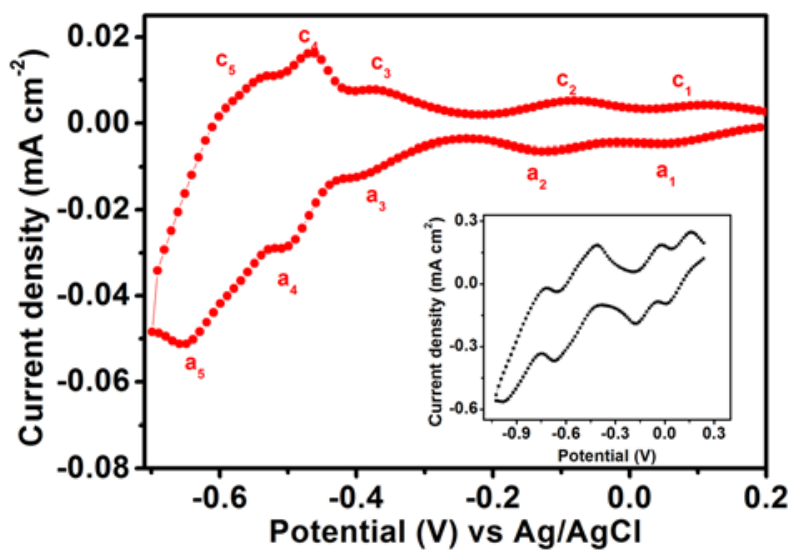

Fig. 3 CV curves of $\mathrm{P}_{2} \mathrm{~W}_{18}$-CNTs multilayer film at a scan rate of $50 \mathrm{mV} / \mathrm{s}$. Inset: $\mathrm{CV}$ of $\mathrm{P}_{2} \mathrm{~W}_{18}$ solution with a scan rate of $50 \mathrm{mV} / \mathrm{s}$.

Galvanostatic charge-discharge tests were employed to evaluate the electrochemical capacitive performance of the composite films. Fig. 4 displays the galvanostatic charge-discharge curves of the electrode based on $\mathrm{P}_{2} \mathrm{~W}_{18}$-CNTs film examined in the voltage window ranged from 0 to $0.5 \mathrm{~V}$ at a constant current of $0.01 \mathrm{~mA} \mathrm{~cm}^{-2}$. It is found that all the curves are not ideal straight line, which is related to the faradic reactions in the process of charge and discharge. The specific capacitance value of the $\mathrm{P}_{2} \mathrm{~W}_{18}$-CNTs film is $0.38 \mathrm{mF} \mathrm{cm}^{-2}$.

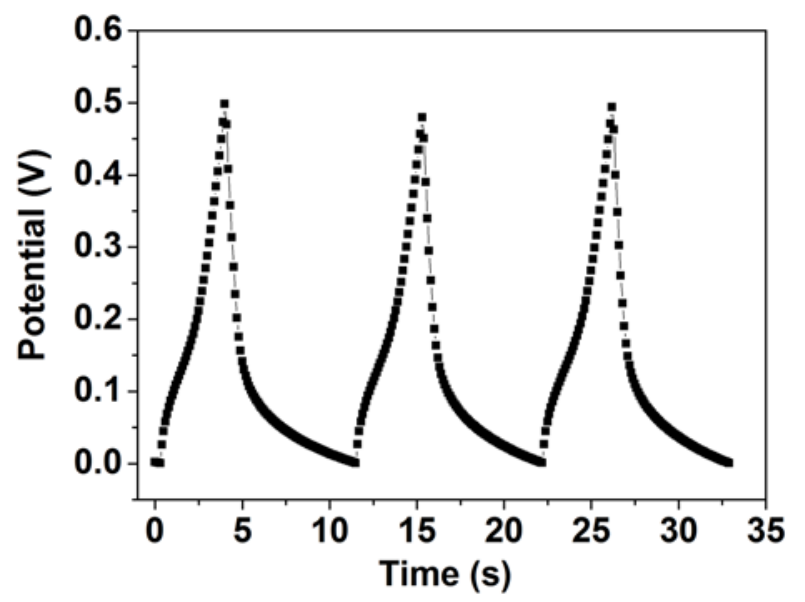

Fig. 4 Galvanostatic charging-discharging of the $\mathrm{P}_{2} \mathrm{~W}_{18}$-CNTs film at a constant current of $0.01 \mathrm{~mA} \mathrm{~cm}^{-2}$ in $0.1 \mathrm{M} \mathrm{H}_{2} \mathrm{SO}_{4}$ electrolyte. 
The cyclic stability of the electrode was evaluated by galvanostatic charge-discharge tests with three-electrode system at the constant current of $0.02 \mathrm{~mA} \mathrm{~cm}^{-2}$. After the first 50 cycles, the specific capacitance of $\mathrm{P}_{2} \mathrm{~W}_{18}$-CNTs film reduced to $95.7 \%$ of its initial specific capacitance. At the 100th cycle, the specific capacitance is $0.272 \mathrm{mF} \mathrm{cm}$, which corresponds to $71.7 \%$ of the initial capacitance value.

\section{Conclusion}

New polyoxometalate-based electrochromic films of $\mathrm{P}_{2} \mathrm{~W}_{18}$-CNTs were successfully fabricated by the LbL self-assembly method. The composite film presents high redox activity and electrochemical capacitive performance by incorporation of CNTs into the film. The composite film is exploited as an electrode for electrochemical supercapacitors with energy density provided by electric double-layer of CNTs and redox processes of POMs, leading to high specific capacitance values. Therefore, the nanocomposite film becomes a promising candidate for possible application in electrochemical supercapacitors.

\section{Acknowledgement}

This work was financially supported by the Natural Science Foundation of China (Grant No. 21301041 and No. 31201376) and Harbin University of Commerce Doctor Start-up Fund Research (No. 12DW030).

\section{References}

[1] Yang Fan, Pei-Fang Liu, Zi-Juan Yang, Tong-Wu Jiang, Kai-Li Yao, Ran Han, XIn-XIn Huo, Yun-Yi Xiong. Relevance Date Relevance - selectedAll access types All access typesOpen Access articlesOpen Archive articles Bi-functional porous carbon spheres derived from pectin as electrode material for supercapacitors and support material for Pt nanowires towards electrocatalytic methanol and ethanol oxidation [J]. Electrochimican Acta, 2015 (163) 140-148.

[2] Selvaraj Chinnathambi, Malika Ammam. A molecular hybrid polyoxometalate-organometallic moieties and its relevance to supercapacitors in physiological electrolytes [J]. Journal of Power Sources, 2015 (284) 524-535.

[3] Yue Zhou, Mehdi Ghaffari, Minren Lin, Ethan M. Parsons, Yang Liu, Brian L. Wardle, Q.M. Zhang. High volumetric electrochemical performance of ultra-high density aligned carbon nanotube supercapacitors with controlled nanomorphology [J]. Electrochimican Acta, 2013 (111) 608-613.

[4] Qing Zhang, Chuan Cai, Jinwen Qin, Bingqing Wei. Tunable self-discharge process of carbon nanotube based supercapacitors [J]. Nano Energy, 2014 (4) 14-22.

[5] Shuping Liu, Lin Xu, Fengyan Li, Weihua Guo, Yan Xing, Zhixia Sun. Carbon nanotubes-assisted polyoxometalate nanocomposite film with enhanced electrochromic performanc[J]. Electrochimica Acta. 2011 (56) 8156-8162.

[6] Shuping Liu, Lin Xu, Fengyan Li, Bingbing Xu, Zhixia Sun. Enhanced electrochromic performance of composite films by combination of polyoxometalate with poly(3,4-ethylenedioxythiophene) [J]. Journal of Materials Chemistry. 2011 (21) 1946-1952.

[7] Elham Nikbakht, Bahram Yadollahi, Mostafa Riahi Farsani. Green oxidation of alcohols in water by a polyoxometalate nano capsule as catalyst [J]. Inorganic Chemistry Communications. 2015 (55) 135-138.

[8] Weiming Wu, Teng Teng, Xiao-Yuan Wu, Xuejing Dui, Lei Zhang, Jinhua Xiong, Ling Wu, Can-Zhong Lu. A cobalt-based polyoxometalate catalyst for efficient visible-light-driven H2 evolution from water splitting [J]. Catalysis Communications. 2015 (64) 44-47. 